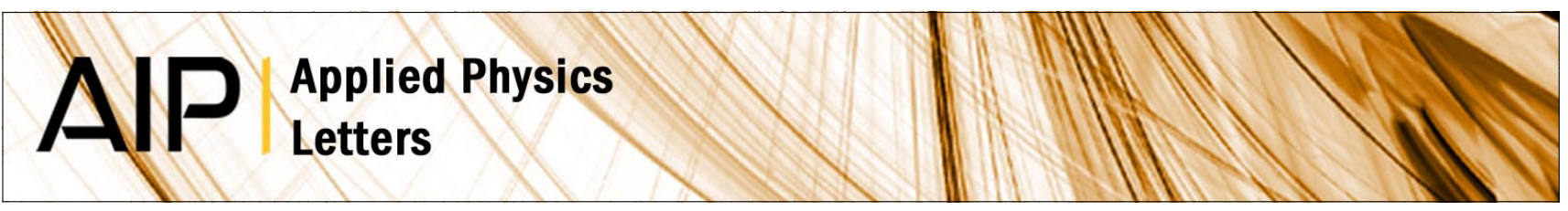

\title{
Detecting squeezed phonons through an indirect radiative transition
}

Ö. E. Müstecaplıoglu and A. S. Shumovsky

Citation: Appl. Phys. Lett. 70, 3489 (1997); doi: 10.1063/1.119209

View online: http://dx.doi.org/10.1063/1.119209

View Table of Contents: http://apl.aip.org/resource/1/APPLAB/v70/i26

Published by the American Institute of Physics.

Additional information on Appl. Phys. Lett.

Journal Homepage: http://apl.aip.org/

Journal Information: http://apl.aip.org/about/about_the_journal

Top downloads: http://apl.aip.org/features/most_downloaded

Information for Authors: http://apl.aip.org/authors

\section{ADVERTISEMENT}

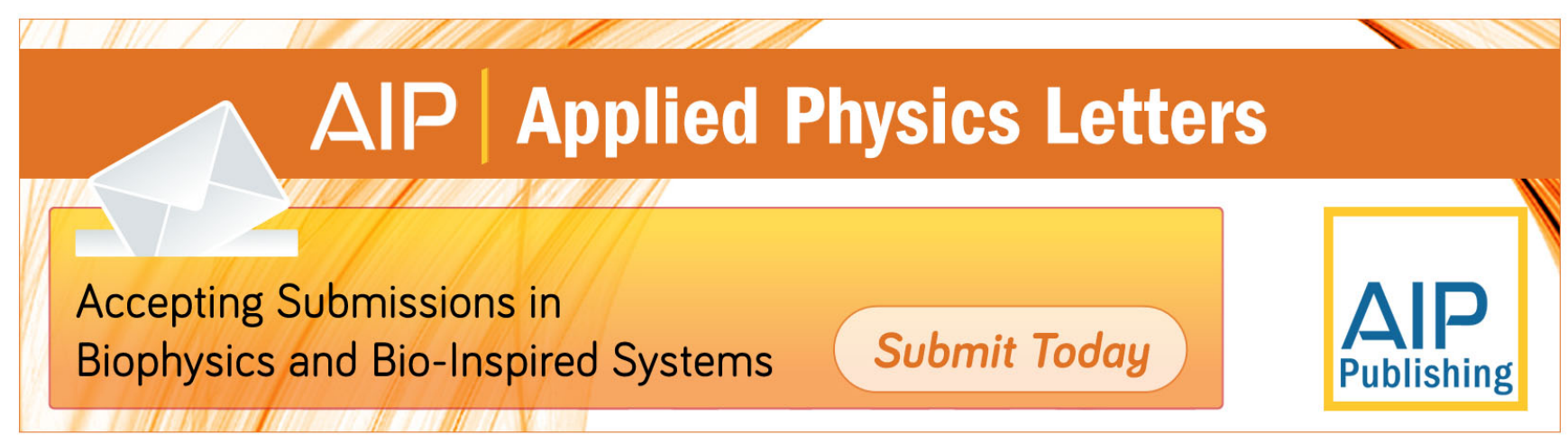




\title{
Detecting squeezed phonons through an indirect radiative transition
}

\author{
Ö. E. Müstecaplıoğlu and A. S. Shumovsky ${ }^{\mathrm{a})}$ \\ Physics Department, Bilkent University, Ankara 06533, Turkey
}

(Received 6 December 1996; accepted for publication 27 April 1997)

\begin{abstract}
A model of the effect of the longitudinal optical phonon number distribution on the Rabi oscillations of the photons involved in the associated indirect transition in a semiconductor is presented. It is shown that a faster cavity photon revival rate is obtained given an initial squeezed thermal phonon state. This is the result of pairwise correlations of phonons due to squeezing and also holds true when phonons are in squeezed vacuum or in squeezed number states. It may be possible to use this effect to detect squeezing in phonons. (C) 1997 American Institute of Physics.

[S0003-6951(97)01326-0]
\end{abstract}

It is known that the longitudinal optical (LO) phonons play an important role in the formation of the photoluminescence spectrum in the case of indirect radiative transitions. An example is provided by the GaAs/AlAs superlattices. ${ }^{1-3}$ Due to the band structure, the lowest electronic states of the conduction band ( $X$ states) are located in the AlAs layers and can be populated by means of the $\Gamma-X$ transfer from the $\Gamma$ states in the GaAs which have been excited initially by laser pulses close to the resonance with the transition $|0\rangle \leftrightarrow|\Gamma\rangle$. It is plausible that the $\Gamma-X$ transfer is dominated by the LOphonon emission. ${ }^{1,2}$ As far as the level $|X\rangle$ is populated, the indirect transition occurs and adds an extra peak to the luminescence spectrum. ${ }^{1-3}$

Obviously, the photons created by the type-II $(|X\rangle \leftrightarrow|0\rangle)$ transition are correlated with the LO phonons and therefore they should take away some information about the phonon number distribution (PND) which is not necessarily the Bose-Einstein distribution (BED) even at thermal equilibrium. ${ }^{4}$ Among these nonclassical PND, the squeezed thermal distributions have attracted great deal of attention. In particular, the nonclassical properties of polaritons have been examined. ${ }^{5-7}$ The possibility of reduction of the fluctuations of the polariton electromagnetic field component ${ }^{5}$ as well as the atomic displacements ${ }^{7}$ has been examined and interesting schemes of measurements have been proposed. In the system under consideration, the phonon-phonon correlations may result from the cascade type transition $|\Gamma\rangle \rightarrow|X\rangle \rightarrow|0\rangle$ with creation of two phonons. Another promising possibility will be briefly mentioned at the end of the article.

In this letter, we examine the effect of the PND on the Rabi oscillations of cavity photons involved in the type-II transition. Rabi oscillations can be observed by the standard methods of quantum optics. ${ }^{8}$ We choose the system as a single type-II transition in a lossless cavity. Then, the problem under consideration formally coincides with the JaynesCummings model with a two-boson transition ${ }^{9}$ described by the Hamiltonian

$$
\begin{aligned}
H= & \omega a^{\dagger} a+\Omega b^{\dagger} b+(\omega+\Omega) R_{X X} \\
& +g\left(R_{X 0} a b+a^{\dagger} b^{\dagger} R_{0 X}\right),
\end{aligned}
$$

where the operators $a, b$ describe the photons and phonons with the frequencies $\omega, \Omega$ respectively, $\left.R_{i j} \equiv \| i\right\rangle\langle j \|$ defines

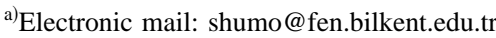

the electron transitions, and $g$ is the coupling constant. Since the Schrödinger problem for (1) can be solved exactly, one can determine the time-dependent density matrix of the system as

$$
\begin{aligned}
\rho(t)= & \sum_{n_{a}, n_{b}=0}^{\infty} \sum_{n_{a}^{\prime}, n_{b}^{\prime}=0}^{\infty} \sum_{l, l^{\prime}= \pm} D_{l l^{\prime}}\left(n_{a}, n_{b} ; n_{a}^{\prime} n_{b}^{\prime}\right) \\
& \times \exp \left\{-i t\left[E_{l}\left(n_{a}, n_{b}\right)-E_{l^{\prime}}\left(n_{a}^{\prime}, n_{b}^{\prime}\right)\right]\right\} \\
& \times\left|\psi_{l}\left(n_{a}, n_{b}\right)\right\rangle\left\langle\psi_{l^{\prime}}\left(n_{a}^{\prime}, n_{b}^{\prime}\right)\right|,
\end{aligned}
$$

where the eigenvalues and eigenstates of (1) at given number of photons $n_{a}$ and phonons $n_{b}$ are

$$
\begin{gathered}
\left.E_{ \pm}=\left[\omega\left(n_{a}+1\right)+\Omega\left(n_{b}+1\right) \pm \Omega_{R}\right)\right], \\
\begin{aligned}
\left|\psi_{ \pm}\left(n_{a}, n_{b}\right)\right\rangle= & \frac{1}{\sqrt{2}}\left(|0\rangle\left|n_{a}+1, n_{b}+1\right\rangle\right. \\
& \left. \pm|X\rangle\left|n_{a}, n_{b}\right\rangle\right) .
\end{aligned}
\end{gathered}
$$

Here $\Omega_{R} \equiv \sqrt{g^{2}\left(n_{a}+1\right)\left(n_{b}+1\right)}$ is the Rabi frequency of the transition. The matrix elements $D_{l l^{\prime}}(\cdot)$ are determined by the initial conditions.

We will suppose that initially the electron subsystem is prepared in the $X$ state, cavity photons are in a coherent state $|\alpha\rangle$, while the phonons have either (BED) or squeezed thermal state ${ }^{10}$ distribution (STD). It is natural to consider phonons as a thermal input field to the squeezing device which is the medium providing the pairwise correlations between phonons. Squeezed thermal state is the sum of the Bose-Einstein weighted squeezed number states and distribution of phonons in that state is pairwise oscillatory. We must emphasize that this behavior is typical for all squeezed state distributions and will be exploited as the signature of squeezing that we shall seek in the radiation from the type-II transition. Hence, we take 


$$
\begin{aligned}
& \rho(0)=(|X\rangle\langle X|) \times(|\alpha\rangle\langle\alpha|) \times \rho_{b}^{(r)}, \\
& \rho_{b}^{(r)}=\sum_{n_{b}=0}^{\infty} P\left(n_{b}\right) S(r)\left|n_{b}\right\rangle\left\langle n_{b}\right| S^{\dagger}(r), \\
& S(r)=\exp \left(\frac{r}{2}\left(a^{2}-a^{\dagger 2}\right)\right), \quad P\left(n_{b}\right)=\frac{\bar{n}_{b}}{\left(\bar{n}_{b}+1\right)^{n_{b}+1}}, \\
& \bar{n}_{b}=\frac{1}{\exp (\Omega / T)-1} .
\end{aligned}
$$

Here $T$ is the temperature and $S(r)$ is the squeezing operator with the real squeezing parameter $r$. The case of BED is provided by $r=0$. These initial conditions completely determine $D_{l l^{\prime}}(\cdot)$ in (2). Then, for the intensity of light generated by the type-II transition we get

$$
I=\operatorname{Tr}\left[\rho(t) a^{\dagger} a\right]=\bar{n}_{a}+\sum_{n_{a}, m} p\left(n_{a}\right) \eta(m) \sin ^{2} \Omega_{R} t,
$$

where $\bar{n}_{a}=|\alpha|^{2}$ is the mean number of photons for the Poisson distribution $p\left(n_{a}\right)$ and, $\eta(m)=\left\langle m\left|\rho_{b}^{(r)}\right| m\right\rangle$ is the STD with mean $\bar{m}$. Since $p\left(n_{a}\right)$ has a sharp peak at $\bar{n}_{a}$, we can apply the method of steepest descent ${ }^{11}$ to perform the summation over $n_{a}$. Then, the intensity takes the form

$$
I=\bar{n}_{a}+\frac{1}{2}-\frac{1}{2} \sum_{k, m=0}^{\infty} h_{k} \eta(m) \exp \left(-\bar{n}_{a} \psi_{k} \epsilon_{k m}^{2}\right) \cos \phi_{k m},
$$

where

$$
\begin{aligned}
& h_{k}=\left(1+k^{2} \pi^{2}\right)^{-1 / 4}, \quad \psi_{k}=2 h_{k}^{4}, \\
& \epsilon_{k m}=g t \sqrt{m+1} / 2|\alpha|-k \pi
\end{aligned}
$$

and

$$
\begin{aligned}
\phi_{k m}= & \bar{n}_{a}\left(2 k \pi+4 \epsilon_{k m}+k \pi \psi_{k} \epsilon_{k m}^{2}\right)-\frac{1}{2} \tan ^{-1}(k \pi) \\
& +\psi_{k} \epsilon_{k m} .
\end{aligned}
$$

Thus, the envelope functions of oscillations in (3) are the Gaussians whose peaks are located at $2 k \pi \sqrt{\bar{n}_{a} /(m+1)} / g$. In the following discussions we use rescaled time $\kappa=t / 2 \pi \alpha$. If there is no phonon contribution, the first revival occurs at $\kappa=1$. The presence of phonons leads to oscillations in the collapse region between $\kappa=0$ and $\kappa=1$. The magnitude of these oscillations increases with $\bar{m}$. In the case of BED, the contribution of phonons into the oscillations vanishes at low temperatures (Fig. 1). In fact, estimating $\Omega \sim 0.02 \mathrm{eV}$ from the half-distance between two peaks in the luminescence spectrum, ${ }^{2}$ we get $\bar{n}_{b} \sim 10^{-13}$ at $10 \mathrm{~K}$ which is typical temperature of measurements. ${ }^{1-3}$ At the same time, STD determines $\bar{m} \sim r$ at low temperature. ${ }^{10}$ Therefore, the oscillations at $0<\kappa<1$ can be observed even at low $T$ if $r$ is high enough. For example, at quite temperate squeezing with $r=1$, the magnitude of oscillations in the collapse region is of the order of half amplitude of the first revival (Fig. 1). A similar effect occurs at the room temperature in the case of BED. However, in this case the peak of the envelope function of that additional oscillation is at $\kappa=2^{-1 / 2}$. This is much closer to the position of first revival $(\kappa=1)$ while in the case of STD the additional peak occurs at $\kappa=3^{-1 / 2}$, which is

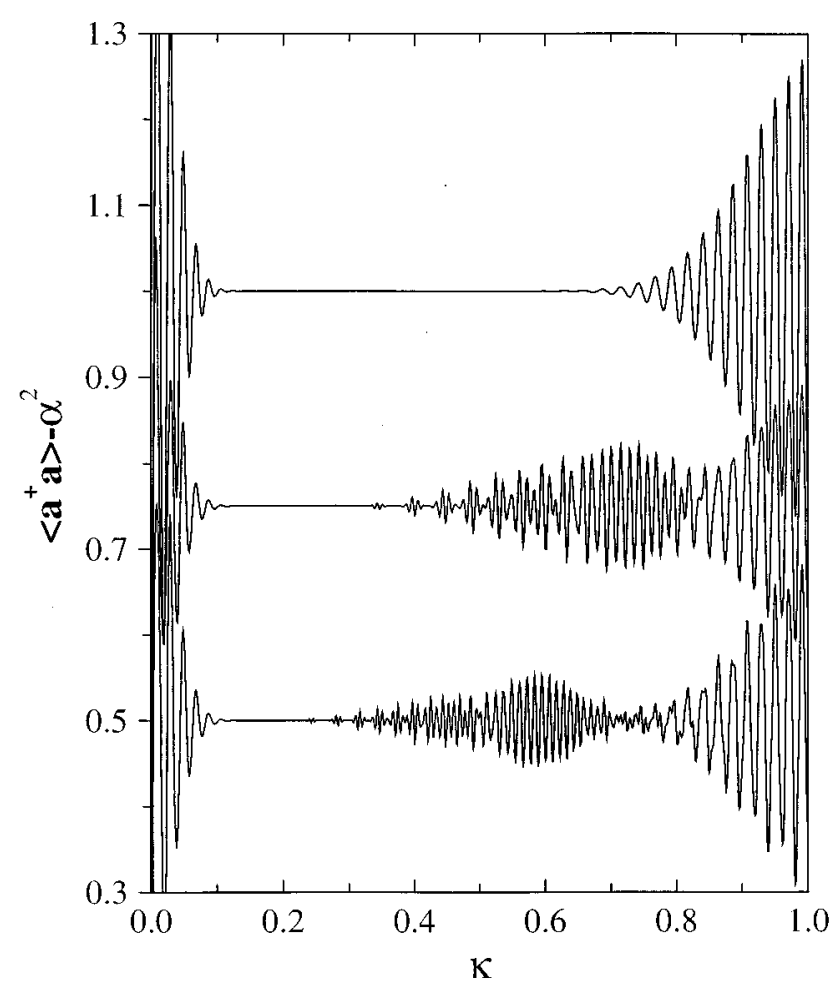

FIG. 1. Rabi oscillations of the cavity photons. Upper, middle and lower graphs correspond to BED at $10 \mathrm{~K}, \mathrm{BED}$ at $300 \mathrm{~K}$, STD with $r=1$ at $10 \mathrm{~K}$, respectively. Upper and the middle graphs are shifted by 0.25 and 0.5 , respectively, for illustration purposes.

close to the midpoint between $\kappa=0$ and $\kappa=1$. The reason is that due to the pairwise nature of STD, the peak position is determined by even $m$ integers while for BED the odd $m$ contribute as well. From Eq. (3), we estimate the amplitude of the peak at $3^{-1 / 2}$ as $0.5\left[1+h_{2} \eta(2)\right] \approx 0.5562$ and also for the peak at 1 (the first revival) as $0.5\left[1+h_{1} \eta(0)\right] \approx 0.682$ in a good agreement with the numerical results (Fig. 1). In general, at any given $k$ one can find an integer $m=3 k^{2}-1$ such that the enveloping Gaussian will be peaked at $3^{-1 / 2}$. Thus, our estimation may be improved by taking into account the contribution of such terms as long as the resulting integers $m$ are even.

The above consideration shows that the presence of squeezing in phonons may be detected by the investigation of the Rabi oscillations of the cavity photons interacting with such phonons through an indirect transition. The question how to prepare phonons in such nonclassical states deserves a separate careful treatment. Nevertheless, we can mention here a promising way. It is possible to make one type of transition dominating the other in short period superlattices by adjusting the laser energy and excitation density. ${ }^{2}$ When type-I recombination is dominant, the system will behave effectively like a $\Lambda$-shaped three level atom. Then the phonon squeezing may be provided by the two phonon transitions between upper $\Gamma-X$ levels if their separation is about $40 \mathrm{meV}$ which is twice the LO phonon energy $\sim 20 \mathrm{meV}$. After that by making the dominating transition type-II, our detection schema should work. Considering the analogies be 
tween three-level quantum optical models for generation of squeezed light and GaAs/AlAs superlattice structures the authors hope that their work will inspire and stimulate the ongoing search for nonclassical phonon states.

${ }^{1}$ J. Feldmann, J. Nunnenkamp, G. Peter, E. Göbel, J. Kuhl, K. Ploog, P. Dawson, and C. T. Foxon, Phys. Rev. B 42, 5809 (1991).

${ }^{2}$ P. W. M. Blom, C. Smit, J. E. M. Haverkort, and J. H. Wolter, Appl. Phys. Lett. 62, 2393 (1993).

${ }^{3}$ F. Agulló-Rueda, H. T. Grahn, and K. Ploog, Phys. Rev. B 49, 14456 (1994).

${ }^{4}$ A. Shumovsky, in Studies in Classical and Quantum Nonlinear Optics, edited by O. Keller (Nova, New York, 1995).
${ }^{5}$ M. Artoni and J. L. Birman, Phys. Rev. B 44, 3736 (1991); Opt. Commun. 104, 319 (1994).

${ }^{6}$ A. Chizhov, R. Nazmitdinov, and A. Shumovsky, Quantum Opt. 3, 1 (1991); Mod. Phys. Lett. B 19, 1233 (1993).

${ }^{7}$ X. Hu and F. Nori, Phys. Rev. Lett. 76, 2294 (1996); Phys. Rev. B 53, 2419 (1996).

${ }^{8}$ Coherence, Amplification, and Quantum Effects in Semiconductor Lasers, edited by Y. Yamamoto (Wiley, New York, 1991).

${ }^{9}$ F. L. Kien and A. S. Shumovsky, Int. J. Mod. Phys. B 5, 2287 (1991); R. Shore and P. L. Knight, J. Mod. Opt. 40, 1195 (1993).

${ }^{10}$ M. S. Kim, F. A. M. de Oliveira, and P. L. Knight, Opt. Commun. 72, 99 (1989).

${ }^{11}$ H.-I. Yoo, J. J. Sanchez-Mondragon, and J. H. Eberly, J. Phys. A 14, 1383 (1981). 